\title{
Cortical closed-loop brain-machine interface requires biomimetic sensory feedback
}

\section{Authors}

A. Abbasi ${ }^{1 \dagger}$, L. Estebanez $^{1 \dagger}$, D. Goueytes ${ }^{1}$, H. Lassagne ${ }^{1}$, D. E. Shulz ${ }^{1 \ddagger}$ and V. Ego-Stengel ${ }^{1 \neq \star}$

Affiliations

${ }^{1}$ Université Paris-Saclay, CNRS, Institut des Neurosciences Paris-Saclay (NeuroPSI), 91190, Gif-sur-Yvette, France.

${ }^{*}$ Correspondence: valerie.ego-stengel@cnrs.fr

${ }^{\dagger}$ Equal contribution.

${ }^{\ddagger}$ Equal contribution.

\section{Summary}

New and improved neuroprosthetics offer great hope for motor-impaired human patients to regain autonomy. One obstacle facing current technologies is that fine motor control requires near-instantaneous somatosensory feedback. The way forward is to artificially recreate the rich, distributed feedback generated by natural movements. Here, we hypothesize that incoming sensory feedback needs to follow biomimetic rules in order to be efficiently integrated by motor circuits. We have developed a rodent closed-loop brain-machine interface where head-fixed mice were trained to control a virtual cursor by modulating the activity of motor cortex neurons. Artificial feedback consisting of precise optogenetic stimulation patterns in the primary somatosensory cortex coupled to the motor cortical activity was provided online to the animal. We found that learning occurred only when the feedback had a topographically biomimetic structure. Shuffling the spatiotemporal organization of the feedback prevented learning the task. These results suggest that the patterns of inputs that are structured by the body map present in the primary somatosensory cortex of all mammals are essential for sensorimotor processing and constitute a backbone that needs to be considered when optimizing artificial sensory feedback for fine neuroprosthetic control.

\section{Keywords}

Brain-machine interface, cortical map, topography, motor, somatosensory, barrel cortex, chronic electrophysiological recordings, behavior, optogenetics, closed-loop neuroscience. 


\section{INTRODUCTION}

Accurate limb control requires somatosensory feedback. Local peripheral anesthesia blocking afferent tactile sensation in human participants reduces dexterity and impairs fine motor control of the hand (Johansson and Westling, 1984; Monzée et al., 2003). Similarly, cortical inactivation of somatosensory cortex in animals has profound effects on motor control (Mathis et al., 2017; Brochier et al., 1999). The critical role of somatosensory feedback has also been obvious in studies of somatosensory-deficient patients, who learn to rely extensively on visual feedback, but can never regain normal movements (Chesler et al., 2016, Sainburg et al., 1995, Miall et al., 2018).

10 In the context of brain-machine interfaces, proprioceptive and touch-like feedback originating from the prosthesis could improve its control. Indeed, recent brain-machine interfaces that have incorporated an afferent somatosensory component have achieved faster prosthesis control (Flesher et al., 2019) and elicited texture-like percepts that cannot be obtained through visual feedback alone (O'Doherty et al., 2019). Thus, for building efficient brain-machine interfaces, a widespread hypothesis is that the feedback should convey somatosensory information about ongoing consequences of the motor control (Bensmaia and Miller, 2014). Such artificial tactile information can be provided through direct electrical activation of the cerebral cortex (Armenta Salas et al., 2018; O'Connor et al., 2013; O'Doherty et al., 2011; Romo et al., 1998; Tabot et al., 2013). However, recreating naturalistic sensations through cortical microstimulation raises important challenges.

One major challenge arises from the fact that somatosensation is a distributed sense. Indeed, a percept often results from the integration of multiple inputs from touch sensors spread over large areas of the skin. According to the classical somatotopic representation, separate peripheral areas of the skin are processed by distinct cortical columns (Penfield and Boldrey, 1937). To provide distributed feedback in the context of an invasive brain-machine interface, recent approaches have started to implement the stimulation of several locations in the somatosensory cortex (Flesher et al., 2016; Hartmann et al., 2016). Beyond the technical challenge, this raises the question of how to distribute sensory information onto the cortical surface. One approach, based on biomimicry, consists in using somatotopy to attempt to elicit percepts in locations distributed as during natural sensory experiences. Conversely, the adaptation approach states that animals can be trained to discriminate non-naturalistic patterns of cortical stimulation, thanks to the plasticity of cortical networks (Bensmaia and Miller, 2014).

Here, we hypothesize that biomimetic patterns of cortical feedback stimulations are more likely to be integrated in a brain-machine interface, compared to shuffled patterns of feedback. We tested this hypothesis directly by training mice to control a one-dimensional cursor with motor cortex neuronal activity, while receiving on-line one of several patterns of cortical feedback. This direct input was delivered intracortically by photostimulating the representation of whiskers in the primary somatosensory cortex (S1). This cortical area exhibits a particularly manifest topography, with anatomical structures named barrels corresponding to whiskers on the snout of the animal. Mice were able to learn the task only in a biomimetic feedback condition, when the sensory inputs followed naturalistic patterns, but could not learn it if the sensory feedback was spatially shuffled or if no feedback was provided. 


\section{RESULTS}

\section{Optogenetic S1 feedback enables learning in a closed-loop brain-machine interface}

We trained mice to solve a cortical closed-loop brain-machine interface task. Neuronal activity was recorded in layer 5 of the primary motor cortex (M1) with chronically implanted silicon tetrodes (Figure 1A-B, Figures S1, S2). Specifically, mice had to modulate online the summed firing rate of three neurons recorded simultaneously (Master neurons) to control the location of a virtual cursor among eight possible positions (Figure 1C, Figure S3). When the cursor was in the rewarded position (either position 4, 5 or 6, see Methods), the mice could obtain water by licking a reward port. If the mice licked when the virtual cursor was outside of the rewarded position, no water was provided but no punishment schedule was imposed neither.
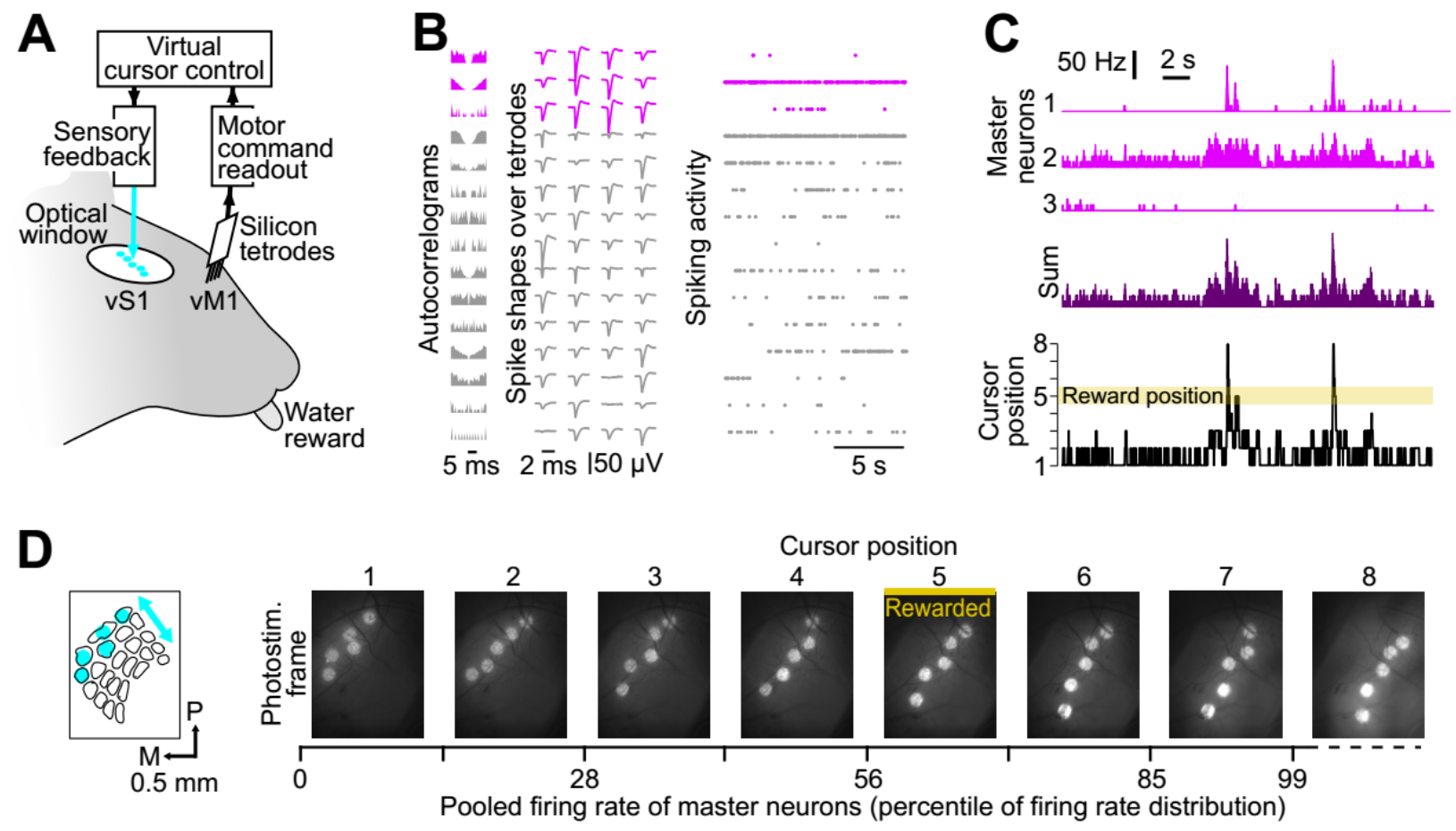

Figure 1. Mice control a virtual cursor with motor cortex activity with online sensory feedback delivered to somatosensory cortex.

(A) Closed-loop setup combining chronic extracellular recording in M1 and optogenetic feedback that targets the barrels in S1.

(B) Tetrode recordings of baseline activity in M1, including spike shapes and autocorrelograms. Purple: Master neurons that are selected to control a virtual cursor. Gray: Neighboring neurons recorded simultaneously.

(C) Top: Time histograms showing the activity of the 3 Master neurons (bins of $10 \mathrm{~ms}$ ) Middle: Sum of the activity of the Master neurons. Bottom: Position of the virtual cursor obtained from the summed activity. The cursor must be in position 5 for the mouse to obtain a reward by licking.

(D) Bar-like naturalistic photoactivation of the S1 barrels. Left: Schematic of the first photostimulation frame. P: posterior, M: medial. Right: Snapshots of the cortical surface illustrating biomimetic photostimulation frames for each cursor position. When the cursor is in position 5 , licks are rewarded. 
During the task, information about the current position of the virtual cursor was made available online through patterned optogenetic stimulation of S1. The mice, which expressed constitutively channelrhodopsin in pyramidal neurons (Emx-Cre;Ai27 strain, Madisen et al., 2012), received photoactivation spots in different locations of the barrel field in the primary somatosensory cortex (S1). The feedback conveyed information about the current position of the cursor with a very short latency $(12 \pm 5 \mathrm{~ms}$, Abbasi et al., 2018). At each time point, the illumination pattern consisted of the simultaneous stimulation of five barrels, corresponding to the dorsoventral arcs of whiskers on the snout. Over time, the optogenetic stimulation mimicked the sequential deflection of whiskers that is produced when a vertical object moves across the mouse snout (Figure 1D) (Jacob et al., 2008; Vilarchao et al., 2018). Thus, the goal of the mouse was to adjust the activity of neurons in the motor cortex such as to bring the cursor to the rewarded position. This position was signaled to the animal by the feedback optogenetic frame, allowing the animal to lick the water spout to obtain water reward when that specific frame was on.
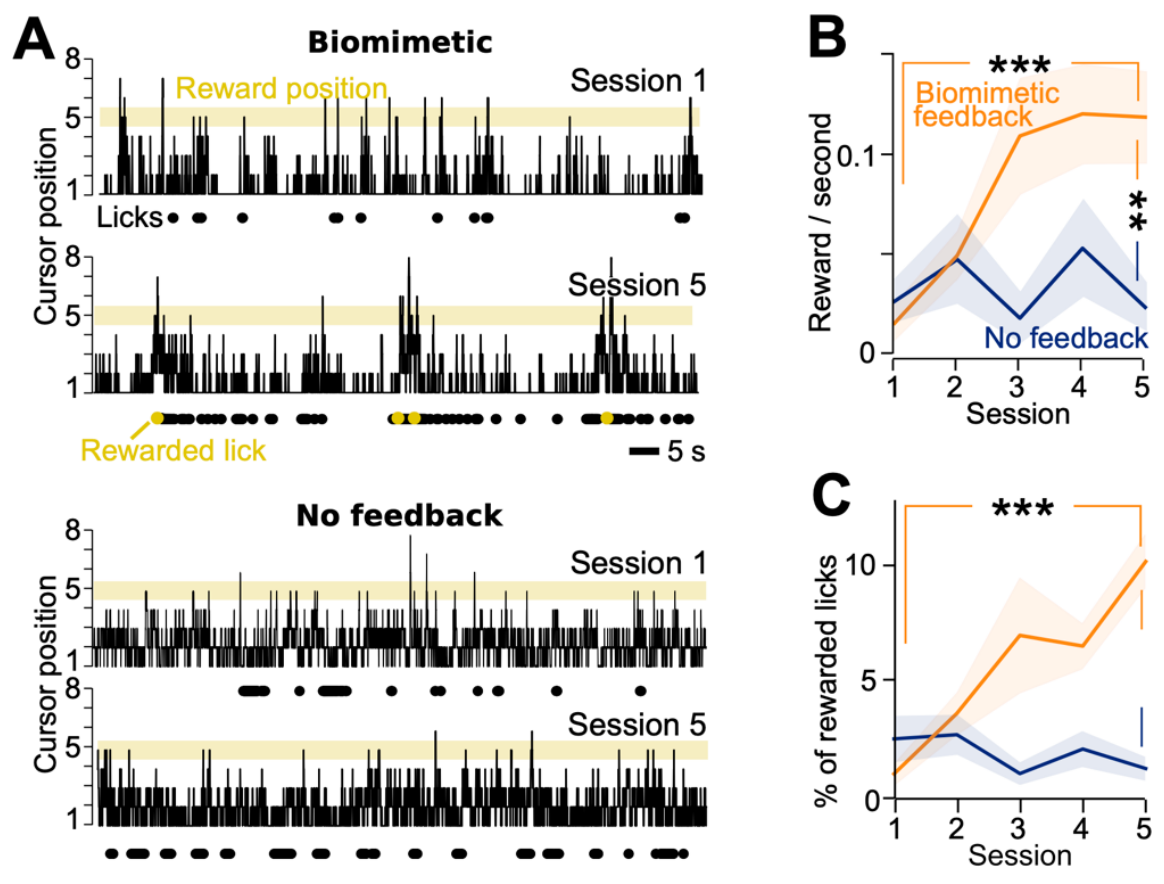

Figure 2. Sensory feedback to the barrel cortex enables learning of a brain-machine interface task.

(A) Example cursor position, licking, and reward time course during first (Session 1) and last (Session 5) training sessions using the biomimetic feedback (Top) or no feedback (Bottom).

$(B, C)$ Frequency of rewards $(B)$ and proportion of licks $(C)$ that were rewarded during learning with biomimetic feedback (orange, 10 mice) versus no feedback (blue, 8 mice). ${ }^{* *}: p<0.01 .{ }^{* * *}: p<0.001$, non-parametric Mann-Whitney tests. Shaded backgrounds: \pm standard error of the mean (SEM).

Disrupting the biomimetic structure of the feedback prevents learning 
Mice were able to learn the task rapidly with this biomimetic cortical feedback. Figure 2A (Left) shows an example in which in the first daily 30-min session, the mouse licked occasionally, but because the cursor was rarely in the reward position during those licks, it obtained no water across the time interval displayed. In the fifth day training session, the same mouse started licking bouts at times when the Master neuron activity was high, and thus obtained rewards more frequently. Indeed, over the course of five training sessions, the frequency of rewards significantly increased more than ten times on average (from 0.014 to 0.19 rewards/s; orange curve of Figure 2B, Mann-Whitney $U=5, p<0.001, n=10$ mice). In contrast, in the absence of optogenetic feedback, mice failed to reliably increase the frequency of rewards despite the same amount of training ( 0.025 vs 0.022 rewards/s; example in Figure $2 \mathrm{~A}$ bottom; blue curve in Figure 2B, Mann-Whitney $U=31, p=0.48, n=8$ mice among the 10 tested in the biomimetic condition).

The increase in reward frequency in the biomimetic feedback condition was accompanied by an increased proportion of licks that were rewarded (Figure $2 \mathrm{C}$, Mann-Whitney $U=1, p<$ 0.001). This indicated that the mice did not simply increase their licking frequency irrespective of the cursor position in order to solve the task. We conclude from this data that sensory feedback to the barrel cortex is required for learning this brain-machine interface task in five daily training sessions.
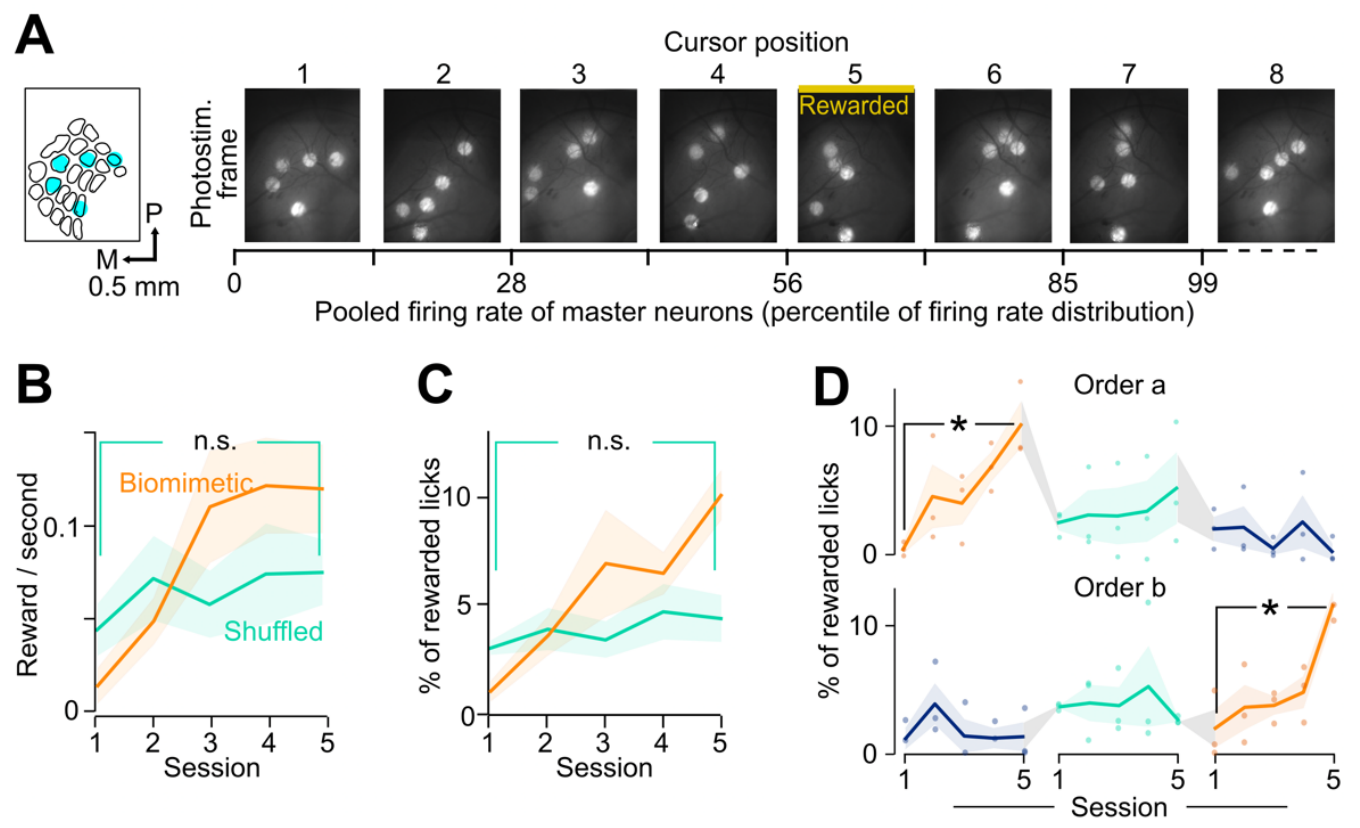

Figure 3. Learning relies on biomimetic topographic feedback.

(A) Left: Schematic of the first shuffled photostimulation frame. Right: Snapshots of the cortical surface illustrating the shuffled photostimulation frame for each cursor position.

(B) Mean reward frequency across training sessions with biomimetic (orange, 10 mice) and shuffled (green, 8 mice) feedback.

(C) Same as (B) for percentage of rewarded licks.

(D) Learning curves for two different orders of presentation of the three protocols: biomimetic (orange), shuffled (green) and no feedback (blue). ${ }^{*}: p<0.05$. Mann-Whitney non-parametric tests. Individual data points are overlaid. For panels $B-D$, shaded backgrounds: \pm SEM. 
Our results showed that the mice could learn the task with biomimetic optogenetic feedback. However, any arbitrary feedback encoding the position of the virtual cursor might suffice to improve task performance. To test this possibility, we trained mice in an additional protocol in which the feedback still carried the online information about the cursor position among the eight possible positions, but where the biomimetic structure of the feedback was spatially scrambled (Figure 3A, shuffled feedback, 8 mice among the 10 tested in the biomimetic condition). In this condition, mice failed to learn the task, an impairment measured both in terms of reward rate (green curve in Figure 3B, Mann-Whitney $U=5, p=0.095$ ) and proportion of rewarded licks (Figure $3 \mathrm{C}$, Mann-Whitney $U=28, p=0.36$ ). Thus, we conclude that a biomimetic topographic feedback was required for learning the task.

The order of the three training conditions (biomimetic, shuffled and no feedback) might have had an impact on the learning performance of the animals. To investigate this, we separated the six mice trained on all three protocols into two sets of three mice, which were trained according to two different orders ( $a$ and b, Figure 3D). Irrespective of the protocol order, significant learning was observed only in the biomimetic feedback condition (Mann-Whitney $U$ $=0, p=0.04$ ). We conclude that the training order did not impact the learning process, and that it is indeed the naturalistic properties of the biomimetic feedback that optimized the learning.

\section{Biomimetic feedback allows accurate motor control}

In order to control the virtual cursor accurately, Master neurons in M1 need to adapt their activity on the basis of the incoming sensory feedback from S1, pooling information spread over several barrels in S1. This online integration by Master neurons could be particularly impacted by the topographical layout of the sensory patterns, notably through nonlinear integration across time as the cursor progresses towards the reward zone. We hypothesize that the naturalistic shift of the optogenetic patterns on the cortical surface of S1 in the biomimetic condition could lead to an optimal integration of the feedback by Master neurons in M1. This would involve an effective modulation of their activity, leading to more accurate cursor dynamics. Note that because of the closed-loop design with very low latencies, the cursor position and the activity of Master neurons display parallel dynamics. To investigate in more depth the cellular mechanisms at play in M1 during learning, we analyzed these dynamics in time and in relation to reward and licking activity.

First, consistent with our hypothesis of differential integration across feedback conditions, we observed significant differences in the neuronal activity between the biomimetic and shuffled conditions, and in particular an increase of the standard deviation of Master neurons' firing rate, exclusively in the biomimetic feedback condition (Figure S4A-B). This increase was not present in simultaneously recorded neighbor neurons. It was accompanied by a significant increase of the total time spent by the cursor in the rewarded position, from $2.4 \%$ in the first session to $3.9 \%$ in the fifth one (Mann-Whitney $U=10, p=0.037, n=6$ mice), which was not present for shuffled stimulations $(1.1 \%$ to $1.7 \%$ from first to fifth session, Mann-Whitney $U=$

$40 \quad 10, p=0.13, n=6$ mice).

The increase in time spent in the rewarded position could result either from a higher variability of mean firing rate (and thus of cursor position) distributed randomly across time, or from an improved neuronal control of the cursor, as seen on the example of Figure 2A. To test this, we focused at a finer time scale on cursor movements (resulting from modulation of the firing rate of neurons in the motor cortex) that took place just before a rewarded lick. In the 
biomimetic feedback condition, one second before the lick, the cursor was significantly closer to the reward position after learning compared to during learning (Figure 4A). The cursor then moved up progressively until it reached the reward position. These smooth dynamics, which were not present when the feedback patterns were shuffled, suggest that the animal learnt to actively control the cursor across time. Furthermore, we observed a significant increase of the mean duration spent in the reward position once entered, from $139 \mathrm{~ms}$ to $246 \mathrm{~ms}$ in a $2 \mathrm{~s}$ window around reward time (Figure 4B). This confirmed the better control of neuronal activity of Master neurons and consequently of the cursor rather than just a random increase in the variability of mean firing rate. The more accurate control of Master neuronal activity resulted in more opportunities to receive rewards.

A
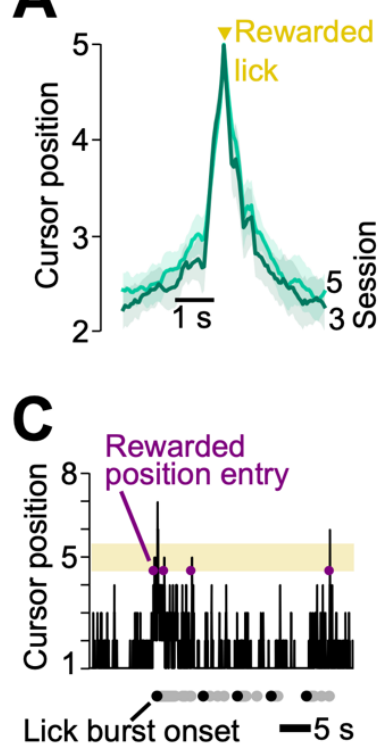
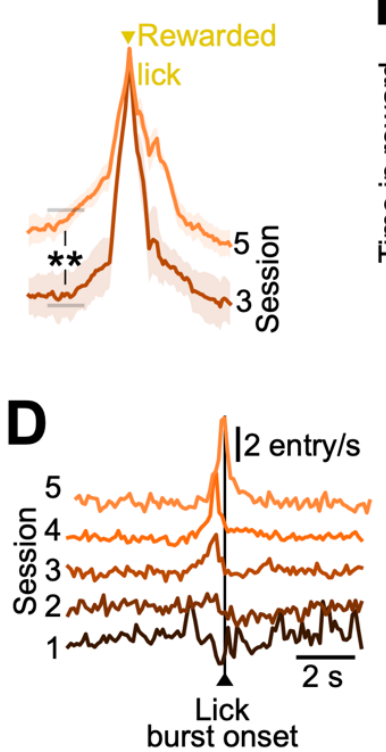
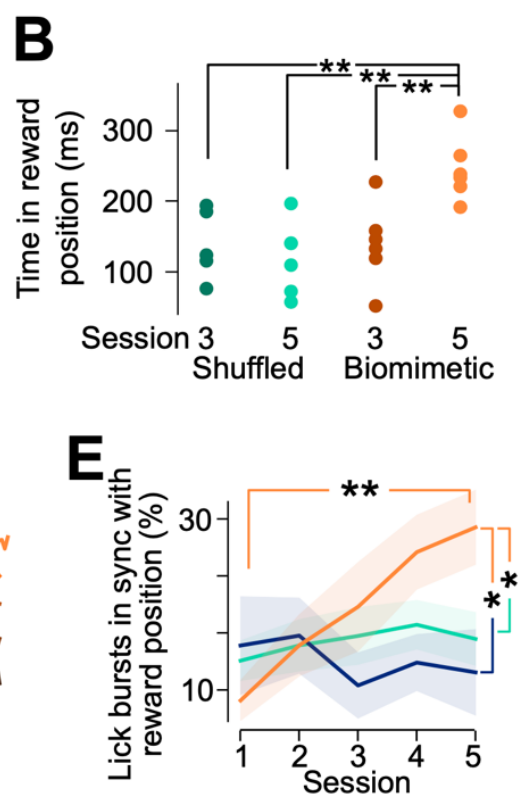

Figure 4. Learning with biomimetic feedback shapes cursor dynamics and licking behavior.

(A) Trajectory of the cursor aligned to the reward time, averaged across the 6 mice where position 5 was rewarded, in the shuffled (Left) and the biomimetic (Right) conditions. Dark colors: session 3. Light colors: session 5. Shaded backgrounds: \pm SEM. ${ }^{* *}$ : Mann-Whitney, $p<0.01$, comparing average firing rates in a 1s window (Gray bar). We compared session 5 to session 3 due to the lack of rewards in sessions 1 and 2.

(B) Average time spent by individual mice in the rewarded cursor position. ${ }^{* *}$ : Mann-Whitney, $p<0.01$ ( $n=6$ mice).

(C) Example behavior. Gray dots: licks. Black: onset of lick bursts. Purple dots: times of entry of the cursor in the rewarded position. To avoid confusion, rewarded licks are not highlighted.

(D) Population average time histogram of the number of entries of the cursor in the rewarded position around the onset of all lick bursts, for the biomimetic feedback condition, across the five training sessions $(n=10$ mice). Baseline levels were shifted upward for clarity.

(E) Percentage of licks bursts that are synchronous (within $\pm 100 \mathrm{~ms}$ ) with entry of the cursor in the rewarded position. Orange: biomimetic $(n=10$ mice); Green: shuffled $(n=8)$; Blue: no feedback $(n=$ 8). *: Mann-Whitney, $p<0.05 .{ }^{* *}: p<0.01$. Shaded backgrounds: \pm SEM. 
In order to obtain rewards, the animal needed to time its licking activity to the availability of reward, that is, to entries of the cursor in the reward zone. Indeed, lick patterns evolved during the course of training (see example in Figure $2 \mathrm{~A}$ ). We first quantified average licking statistics across sessions. Whereas the licking rate was not reliably modified, we observed a significantly increased variability of lick patterns during biomimetic feedback learning (Figure S4C-D). We wondered if, in combination with changes in the cursor dynamics, this led to a better synchronization of licks with the reward position, as in the example illustrated in Figure $4 \mathrm{C}$. We computed time histograms of the entries of the cursor in the rewarded position, aligned on the onsets of lick bursts (Figure 4D). In the initial sessions of learning there was no synchrony between licking and cursor dynamics (bottom brown curve). By session 5, the animal learnt to generate licking bouts starting on average just after the entry of the cursor in the reward zone (top orange curve). We quantified the peaks of these histograms by measuring the proportion of all lick burst onsets that occurred in synchrony ( $\pm 100 \mathrm{~ms}$ ) with an entry in the rewarded position (Figure 4E). This proportion increased significantly across sessions in the biomimetic condition (Mann-Whitney $U=11, p=0.002, n=10$ ) but not in the shuffled condition (Mann-Whitney $U=27, p=0.32, n=8$ ) nor in the no-feedback condition (Mann-Whitney $U=23, p=0.19, n=8$ ). Together, these results show that the animal progressively learns to control the activity of motor neurons, and consequently to move the cursor towards the reward zone and start a lick burst just after.

A

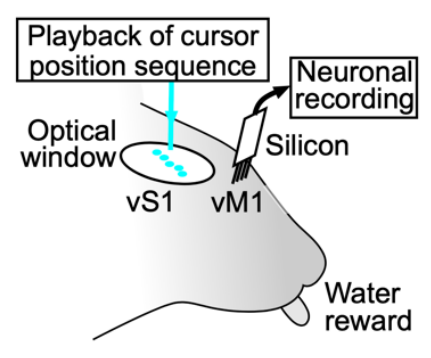

B
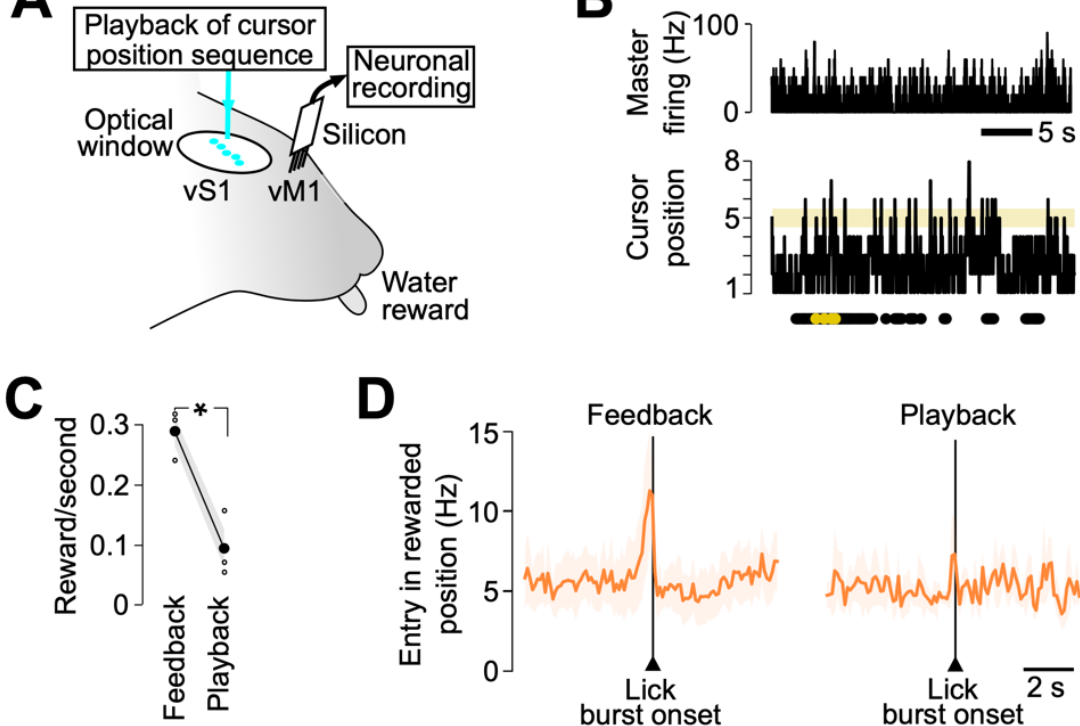

D

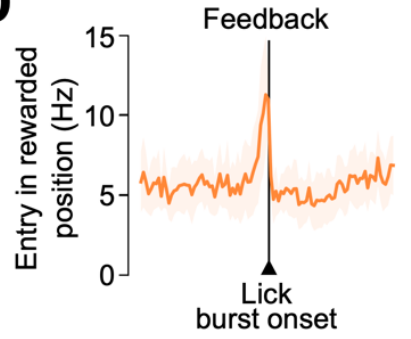

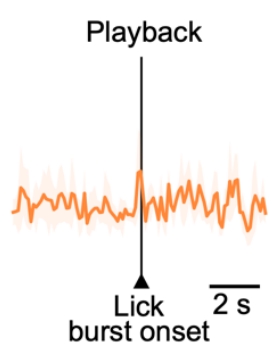

Figure 5. Decoupling cortical stimulation from motor activity impairs performance.

(A) Open-loop configuration with chronic extracellular recording in M1 and optogenetic stimulation on barrels in $\mathrm{S} 1$.

25 (B) Top, time histogram of Master neurons activity during a playback session (bin $=10 \mathrm{~ms}$ ). Bottom, cursor position, licking, and reward time course during the same time interval.

(C) Frequency of rewards during the last session with closed-loop biomimetic feedback and the session with open-loop playback. *: Mann-Whitney, $p<0.05$. Gray background: SEM. $n=3$ mice.

(D) Average histogram of the times of entry of the cursor in the rewarded position around the onset of lick bursts, for the last session with closed-loop biomimetic feedback and the session with open-loop playback. 


\section{Sensory feedback by itself is not sufficient to trigger licking}

Our data show that animals learnt to generate licking bouts around times when the cursor entered the rewarded zone. As emphasized already, in the closed-loop configuration, this corresponds to periods of high Master neurons activity. Thus, we wondered whether the mice learnt to associate reward with increased M1 activity, or whether they learnt to detect the optogenetic pattern signaling reward availability. To test this, we carried out a "playback" session on three mice that had already learnt the full closed-loop task in which M1 activity and optogenetic feedback were decoupled. The mice received the same optogenetic stimulation sequence as in their last session with biomimetic feedback, and could still receive rewards by licking when the cursor was in the rewarded position. However, the cursor dynamics was now independent of the ongoing activity of motor cortex neurons. In other words, the animals were relieved of the motor control aspect of the full task (Figure 5A-B).

Interestingly, the number of rewards per second dropped significantly for each mouse in the playback condition (Figure $5 \mathrm{C}$ ) even though by design, the cursor was as much in the rewarded position as during the biomimetic last session. Analysis of the synchrony between licking onsets and the entries of the cursor in the rewarded position revealed that these events were not coordinated anymore (Figure 5D). From these results, we conclude that the mice did not simply learn to detect the rewarded pattern of optogenetic stimulation. Rather, they learnt to combine both motor control of the cursor and integration of sensory information about its current position to solve the task.

\section{DISCUSSION}

We have shown that sensorimotor integration of artificial feedback stimulations in the context of a cortical brain-machine interface requires a specific spatiotemporal organization of the feedback. Specifically, we found that a biomimetic bar-like stimulation of the barrel cortex could be efficiently integrated by a mouse in order to learn a sensorimotor task. By contrast, a shuffled version of the stimulation, or absence of feedback altogether, prevented learning.

\section{A fast BMI setup for the mouse}

Current research aimed at integrating somatosensory feedback in a cortical brain-machine interface relies on invasive techniques of recording and stimulation in awake behaving animals. Pioneering teams are developing prototypes in non-human primates as well as human participants (Flesher et al., 2019; O'Doherty et al., 2019). Here, we have developed a novel brain-machine interface tailored to the mouse whisker system, a sensorimotor loop that has been described in a very comprehensive way, from the cellular to the network scale (Diamond and Arabzadeh, 2013; Petersen, 2019). This approach has allowed us to take advantage of recent optogenetic tools available for these animals. Thus, we could activate excitatory neurons in the cortex according to spatial light patterns that were adapted, in each individual mouse, to the topographic map of its whiskers present in S1. Furthermore, we benefited from our very low-latency $(10-15 \mathrm{~ms})$ closed-loop design which enables the delivery of feedback online in a dynamic way, that is, while the animal is controlling the movement of the virtual bar. Indeed, a low-latency somatosensory feedback could be an important parameter for optimal sensorimotor learning (Scott, 2016).

\section{Impact of somatosensory feedback on neuroprosthetic learning}

The main result of our study is that learning to control the one-dimensional cursor using motor cortex activity could be achieved over five sessions only when somatosensory feedback was 
provided (biomimetic vs. no-feedback conditions, Figure 2B-C). The direct cortical feedback was updated continuously every $10 \mathrm{~ms}$ throughout the 30 -min sessions. Our results agree with previous studies emphasizing the prevalent role of ongoing sensory feedback in motor learning (Johansson and Flanagan, 2009; Scott, 2016), extending it to neuroprosthetic learning. The animals did not only learn to detect a target, but used the feedback to shape the cursor trajectory over time, demonstrating sensorimotor control (Figure 4).

Importantly, our experimental design did not incorporate a physical implementation of the cursor to be moved by the animal towards the target. Instead, the cursor remained virtual, its dynamics being used only to calculate feedback. This choice ensured that the optogenetic feedback delivered to $\mathbf{S 1}$ was the sole source of sensory information about the cursor position available to the animal during the task. This is in contrast to most previous closed-loop BMI studies, in which ongoing visual feedback of the neuroprosthesis was always available for adjusting motor control (Flesher et al., 2019; O'Doherty et al., 2019). Yet in a recent study, mice were trained to condition motor cortex neurons in an operant way while receiving ratemodulated pulses of optogenetic stimulation in S1, in the absence of visual information (Prsa et al., 2017). After several days, the animals learned to increase the activity of single neurons to obtain reward. Once this behavior was learnt, the animals could still retrieve rewards upon detection of high rates of optogenetic pulses during playback sessions, when motor control was not necessary anymore. This result is at odds with our own findings, in which the mice were unable to maintain their reward rate during the playback sessions (Figure 5). This discrepancy could come from the learning conditions: in the study by Prsa and collaborators, mice did not have to initiate licking to receive reward during learning. This may have contributed to decouple the sensory detection and motor control aspects of the task. Another possibility is that the rich spatiotemporal feedback that we used favored distributed 25 sensorimotor circuits integrating information over large areas of S1, whereas the singlechannel feedback of the above study involved a more restricted set of neurons that became more strongly linked with reward consumption mechanisms.

\section{Biomimetic somatosensory feedback is required for neuroprosthetic learning}

We show for the first time that sensory feedback about the current state of a BMI device, provided directly onto the cortex, should obey spatiotemporal rules of organization of natural input (biomimetic vs. shuffled conditions, Figure 3B-C). In the two conditions, the animal could in theory decode the current position of the cursor from the activation pattern on the cortex. However, only when the sequence of stimulation mimicked that of a bar sweeping across the whiskers, was the animal able to use the information in order to learn to move the cursor progressively and obtain reward. This finding suggests that the sensorimotor task of driving the cursor to the target draws upon pre-existing features of S1-M1 microcircuits linked to their topographic organization (Ferezou et al., 2007). When the topography of the feedback is shuffled, the functional architecture of the cortex is not adapted anymore to the novel sensorimotor computations to be performed. Learning the shuffled task probably requires 40 establishing novel functional connections between sensory input and motor output patterns. We hypothesize that in the shuffled conditions, plasticity mechanisms could allow learning to take place on a longer timescale, supposing that the required connections can indeed be achieved given the existing anatomical scaffold (Fu and Zuo, 2011).

The fact that S1 could not readily adapt to arbitrary patterns of stimulation is in contrast with 45 our results on the motor component of the brain-machine interface. Indeed, consistent with previous publications (Arduin et al., 2013, 2014; Fetz, 1969; Moritz et al., 2008; Prsa et al., 
2017), we found that a few arbitrarily selected M1 neurons could be conditioned in an operant way to learn to control the cursor along one dimension. This difference, between the necessity of biomimetic patterns on the sensory side, and the adaptability of the neuronal networks on the motor side, could have several explanations. One is that we used mesoscale patterns to encode sensory feedback, encompassing large numbers of neurons and connections. Plastic reorganization at this scale could be much more difficult to induce than when targeting only one or a few neurons. Indeed, there is evidence that as the number of neurons controlling motor brain-machine interfaces increases, it becomes necessary to take into account their initial functional connections in order to learn to control the prosthesis rapidly (Athalye et al., 2017; Oby et al., 2019; Sadtler et al., 2014). Another possibility is that primary sensory cortical circuits would be less plastic than motor ones during motor skill learning (Papale and Hooks, 2018). Future experiments will need to address these questions.

\section{Functional role of the $\mathbf{S 1}$ somatotopic map}

So far, the contribution of cortical maps to sensory information processing in general has remained unclear (Kaas, 1997), despite the thorough descriptions of the maps in primary sensory cortices. In the case of the barrel cortex, several of the functional properties encoded by its neurons are spatially organized inside the map beyond spatial topography (Andermann and Moore, 2006; Kremer et al., 2011; Simons, 1978). For example, rodent S1 neurons can be selective to several multi-whisker features such as correlation between whisker movements (Estebanez et al., 2012) and global motion across the snout (Jacob et al., 2008). Correlation selectivity is distributed along a specific gradient from the center of barrels to the septa between barrels (Estebanez et al., 2016), while global motion selectivity obeys a supra-barrel organization (Vilarchao et al., 2018). The large-scale organization of feature encoding would be favored because of the dense lateral connectivity inside S1, enabling distributed cortical computations (Feldmeyer, 2012). Through this rich anatomical substrate, non-linear spatiotemporal integration in $\mathrm{S} 1$ results in enhanced responses to some input patterns, and suppression of responses to other patterns (Estebanez et al., 2012). However, so far, these feature extraction properties have not been causally linked to behavior.

Our results shed light on the functional role of topography of the somatosensory cortical map in the behaving animal, by testing causally the impact of different patterns of sensory input, and in particular by comparing biomimetic versus arbitrary patterns. In our study, mice had to detect patterns of stimulation projected onto a large area of $\mathrm{S} 1$. Only biomimetic spatiotemporal feedback patterns enabled the animal to use the information about the cursor position in order to solve the task. In these conditions, the expected integration of information across barrels could drive the activity of Master neurons in the motor cortex. Hence, our results suggest that the somatotopic organization of $\mathrm{S} 1$ plays a critical role in sensorimotor integration.

Current BMI prototypes require long training and lack precision and flexibility, probably because they lack the appropriate somatosensory feedback (Bensmaia and Miller, 2014).

40 From our results, we propose that feedback strategies based on intracortical stimulation should obey the known topography of somatosensory areas. We hope that unveiling such fundamental constraints of neuronal circuits will enable the development of a new generation of BMls, incorporating rich proprioceptive and tactile feedback essential to achieve dexterity and embodiment. 


\section{METHODS}

\section{Mouse preparation}

All animal experiments were performed according to European and French law as well as CNRS guidelines and were approved by the French ministry for research (ethical committee 59, authorization 858-2015060516116339v5). The data were obtained from 10 adult (6-10 weeks old) Emx1-Cre;Ai27 mice. Detailed procedures for bidirectional cortical interfacing of the mice were described previously (Abbasi et al., 2018). Briefly, all surgeries were performed under isoflurane anesthesia (1\%-4\% Isoflurane depending on mouse state, assessed by breathing rate and response to tail pinch), in 100\% air. For each mouse, during a first surgery, a $5 \mathrm{~mm}$ glass optical window was implanted over the left primary somatosensory cortex (S1, P-1.5 mm and L-3.3 mm, from bregma) and a head-fixation bar was implanted on the contralateral side of the skull. Eight days later, intrinsic imaging was performed during mechanical deflection of at least 3 individual whiskers to locate a subset of barrels in the barrel cortex. In the following days, a second surgery was performed to chronically implant (Okun et al., 2016) a 32 channel silicon probe (A1x32Poly35mm25s 177A32, Neuronexus, USA) in the whisker zone of the motor cortex (M1, A1.5 mm L0.6 mm from bregma, electrode recording sites $650-800 \mu \mathrm{m}$ deep in cortex).

\section{Neuronal electrophysiological recordings}

Following the surgery, mice were monitored during 5 days to ensure stability of the shape and amplitude of the units isolated by the online spike sorting (Blackrock microsystems, USA). Clusters corresponding to well-defined single units (consistent spike shape and adequate autocorrelogram, Figure 1B) were manually selected within the tetrode spike amplitude space. This manual selection was controlled before each session to ensure that we maintained unit separation while keeping track of the same units across sessions (Figure S1). Once units were selected, the training session was initiated and individual spikes were sorted according to the pre-defined clustering. At the start of the training sessions, we recorded a median of 25.5 neurons simultaneously (interquartile range $[I Q R]=5.25$ neurons, $n=10$ mice). After 17 days (average last training session) we recorded a median of 25 neurons (IQR $=16$ neurons, $n=$ 10 mice).

\section{Brain-machine interfacing}

Among the recorded units of each mouse/session, a set of 3 putative pyramidal neurons - the Master neurons - were selected by the operator. In the first two mice, we initially enrolled 7 neurons. Their summed activity was then convolved with a $100 \mathrm{~ms}$ box kernel and its value determined the position of a virtual cursor. Mice were required to control the position of the cursor to solve the task. To do so, the values of the summed firing rate of the Master neurons were recorded during the first 3 minutes that preceded the start of the session. A threshold was set at the 99th percentile of theses baseline activity values, and the activity space from 0 up to this threshold was split in 7 equal positions, with an extra 8th position for values above the 99th percentile threshold. For six mice, the 5th position was rewarded, which means that whenever the cursor was inside that position and the mouse simultaneously licked, it obtained a $5 \mu \mathrm{L}$ water drop (Figure S3). For the other six mice, either the 6th or the 4th position was rewarded. These mice were included in all analyses except the quantification of cursor trajectory around cursor position 5 (Figure 4A-B). 
If the recording of one neuron was lost during the training, the active Neighbor neuron with the largest spike shape was enrolled to replace it. If no additional Neighbor neuron was available, the experiment kept going with a reduced count of Master neurons, down to two neurons in one mouse.

\section{Optogenetic photostimulation of somatosensory cortex}

Each cursor position was associated with a specific feedback pattern that was projected onto the barrel cortex of the mice using a Digital Light Processing module (DLP, Vialux V-7001, Germany). The DLP contained a 1024 x 768 pixels Texas Instruments micro-mirror chip, which was illuminated by a high-power $462 \mathrm{~nm}$ blue LED. The image stream generated by the device was focused onto the cortical optical window using a tandem-lens macroscope (Ceballo et al., 2019). This device was used to send homogeneous light spots, $200 \mu \mathrm{m}$ in diameter, centered onto the barrel locations for the mouse strain/age that we used (Abbasi et al., 2018). A set of reference barrels (at least 3 ) that were located during the mouse preparation via intrinsic imaging were used to align a standard barrel map (Knutsen et al., 2016). We used three different sets of feedback frames to obtain the 3 conditions we tested: biomimetic (Figure 1D), shuffled stimulations (Figure 2A), and finally a condition where no photostimulation was displayed (black frames).

\section{Behavioral training}

Mice were first habituated to head-fixation (1 session). Then, they were water deprived and learned to lick for water from a reward tube in front of their mouth (1-2 sessions) (Guo et al., 2014). Finally, the mice were trained on the 3 different feedback conditions for 5 sessions (1 session/day). Training sessions lasted 30 minutes each. Mice were checked daily for weight and extra water/food intake was provided as needed to stabilize the weight at $80 \%$ of their initial value.

\section{Offline spike sorting}

Offline extraction of neuronal activity was performed using SpyKING CIRCUS (Yger et al., 2018). We confirmed that each online sorted Master unit was properly spike sorted by matching it with a specific offline-sorted unit, by comparing spike shapes and amplitudes across tetrodes. All additional, non-Master offline-sorted units were labeled as Neighbor units.

\section{Data Analysis}

All statistical tests are non-parametric, two-sided Mann-Whitney tests. In Figures 1 and 2 all licks are taken into account. In Figure 3E, lick bursts are defined as series of licks where the inter-lick interval is smaller than $200 \mathrm{~ms}$.

\section{Intracortical microstimulation (ICMS) experiments}

35 To confirm that the electrodes were located in the motor cortical area, we performed ICMS at the end of the behavior sessions ( $n=3$ mice, Figure $S 2)$. We injected bipolar current pulses (amplitude $21 \mu \mathrm{A} /$ channel, duration $1.4 \mathrm{~s}$, frequency $60 \mathrm{~Hz}$ ) through the 32-channel Neuronexus silicon probe implanted in $\mathrm{M1}$, in awake head-fixed animals. The contralateral whiskers were imaged using a high-speed videography (camera - Baumer HXc-20, lens - 6 $\mathrm{mm}, \mathrm{F} / 1.4$ ) at 300 frames per seconds for a duration of $9 \mathrm{~s}$. A single trial consisted of $5 \mathrm{~s}$ preICMS videography, followed by $1.4 \mathrm{~s}$ during ICMS stimulation and finally $2.6 \mathrm{~s}$ post-ICMS. This procedure was repeated 14 times during a single session of ICMS experiment, with a 1 $s$ inter-trial delay. In the ICMS videos, a central whisker was identified amongst all the whiskers 
in the field of view, and tracked using the automated video tracking software DeepLabCut (Mathis et al., 2018). The amplitude of ICMS-evoked whisker movement was defined as the mean whisker angle during the first $1 \mathrm{~s}$ of stimulation versus the $1 \mathrm{~s}$ immediately before. Latency of whisker movement was measured at the first frame with significant whisker movement amplitude (2 standard deviations above the mean).

\section{Histology}

After the experiment, mice were deeply anaesthetized with isoflurane (4-5\%) and pentobarbital, then exsanguinated and perfused with $4 \%$ paraformaldehyde (PFA). The brains were extracted and stored overnight in 4\% PFA. The brains were then transferred to a solution of phosphate-buffered saline for at least 24 hours before $50 \mu \mathrm{m}$ slices in the coronal plane were cut and stained for cytochrome $\mathrm{C}$ oxidase. The location and depth of the silicon probe in the brain were traced by Dil depositing on the electrodes prior to their implantation and by looking afterwards at the fluorescent dye present in the histological slices (Figure S2A).

\section{Acknowledgments}

We thank Isabelle Férézou for advice on experimental design and analysis; Ehud Ahissar, Boris Barbour, Karim Benchenane, Suliann Ben Hamed, Sliman Bensmaia, Daniel Feldman, Evan Harrell and German Sumbre for comments on an earlier version of the manuscript. Aurélie Daret and Guillaume Hucher provided experimental support. FRM (Equipe FRM DEQ20170336761), CNRS (80|Prime), ANR Neurowhisk, Lidex NeuroSaclay, Idex Brainscopes and iCODE, and FRC (AAP 2018) provided funding.

\section{Author contributions}

D.E.S and V.S. obtained funding and administrated the project. D.E.S, L.E and V.S conceptualized and supervised the experiments and analysis; A.A. carried the investigation with support from L.E., H.L. and D.G.; L.E and A.A. analyzed the data with support from H.L.; L.E., V.S. and D.E.S wrote the manuscript with input from all authors.

Declaration of interests: The authors declare no competing interests. 

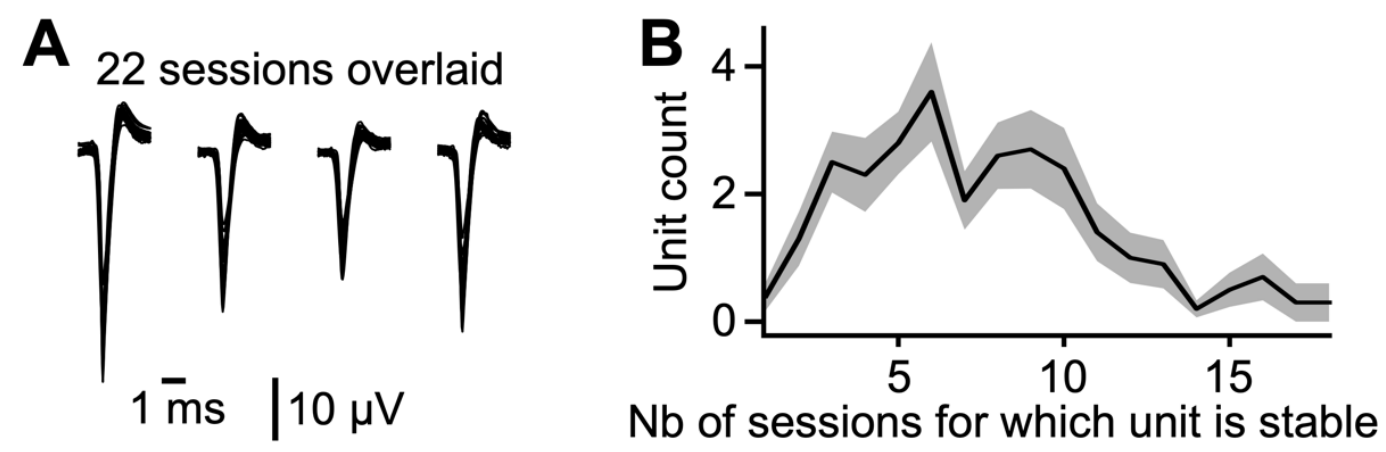

Supplementary Figure 1. Stability of the recorded neurons. (A) Spike shape of a neuron recorded through the four contacts of a tetrode, overlaid over 22 sessions (1 session/day). (B) Average distribution of the count of sessions where the same unit could be identified in the recording, for all units included in the dataset $(n=10$ mice). Shaded background: standard error of the mean. 
bioRxiv preprint doi: https://doi.org/10.1101/2019.12.12.873794; this version posted July 14, 2020. The copyright holder for this preprint (which was not certified by peer review) is the author/funder, who has granted bioRxiv a license to display the preprint in perpetuity. It is made available under aCC-BY-NC-ND 4.0 International license.
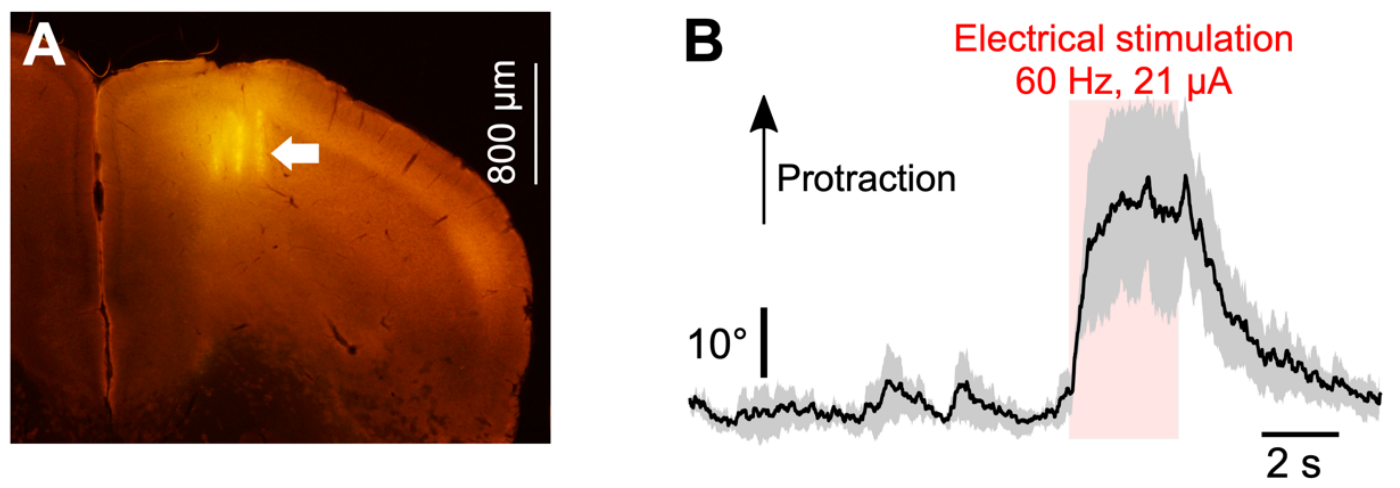

Supplementary Figure 2. Localization of the implanted silicon probes in whisker M1. (A) Coronal slice ( $50 \mu \mathrm{m}$ thick) of a mouse brain, stained for Cytochrome oxidase. Dil coating of the shanks prior to insertion resulted in fluorescent lines indicative of the location of single shanks (yellow tracks) that confirm the location of the silicon tetrode array in M1 (white arrow). (B) The amplitude of angular movements of a contralateral whisker evoked by ICMS stimulation through the silicon probe (average of 3 mice) confirms that the electrode was located in M1 (see Methods). Shaded background: standard error of the mean. 

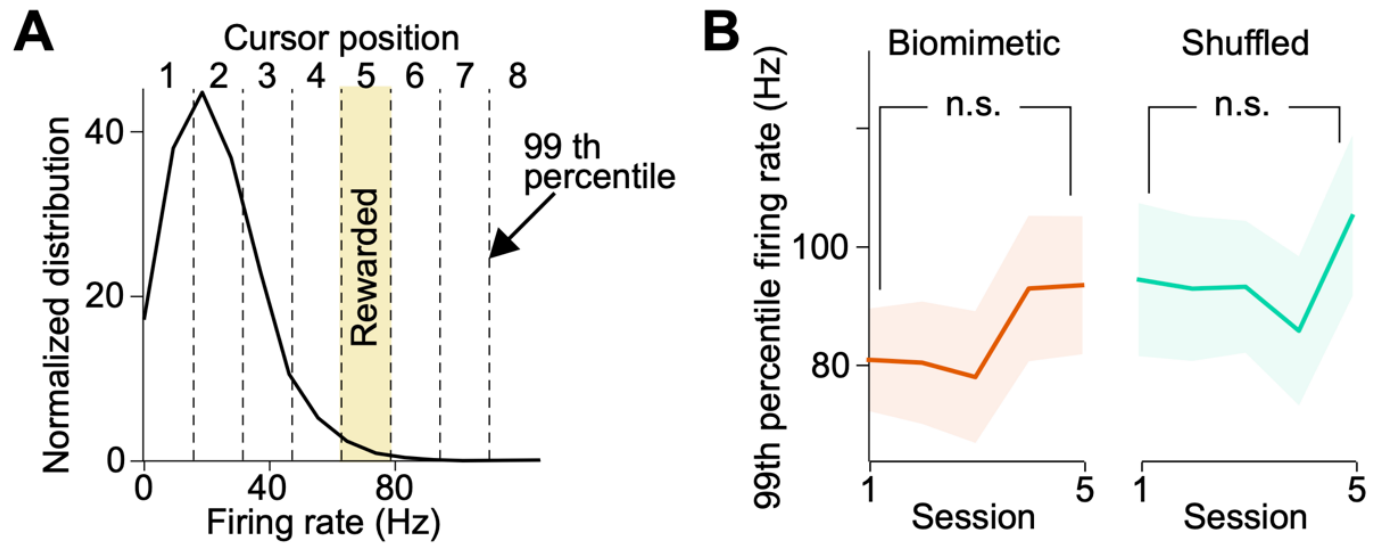

No feedback

Supplementary Figure 3. Definition of the firing rate limits of the positions of the cursor. (A) Example distribution of the firing rate during the 3 min baseline at the start of a session. Positions correspond to equal firing rate intervals. Position 1 starts at $0 \mathrm{~Hz}$ firing rate. The transition between cursor positions 7 and 8 is set at the 99th percentile of the firing rate distribution. (B) The 99th percentile of the firing rate (averaged across mice) did not significantly evolve over training sessions (n.s.: Mann-Whitney $\mathrm{p}$ value $>0.05$ ). Shaded background: +/- SEM. 

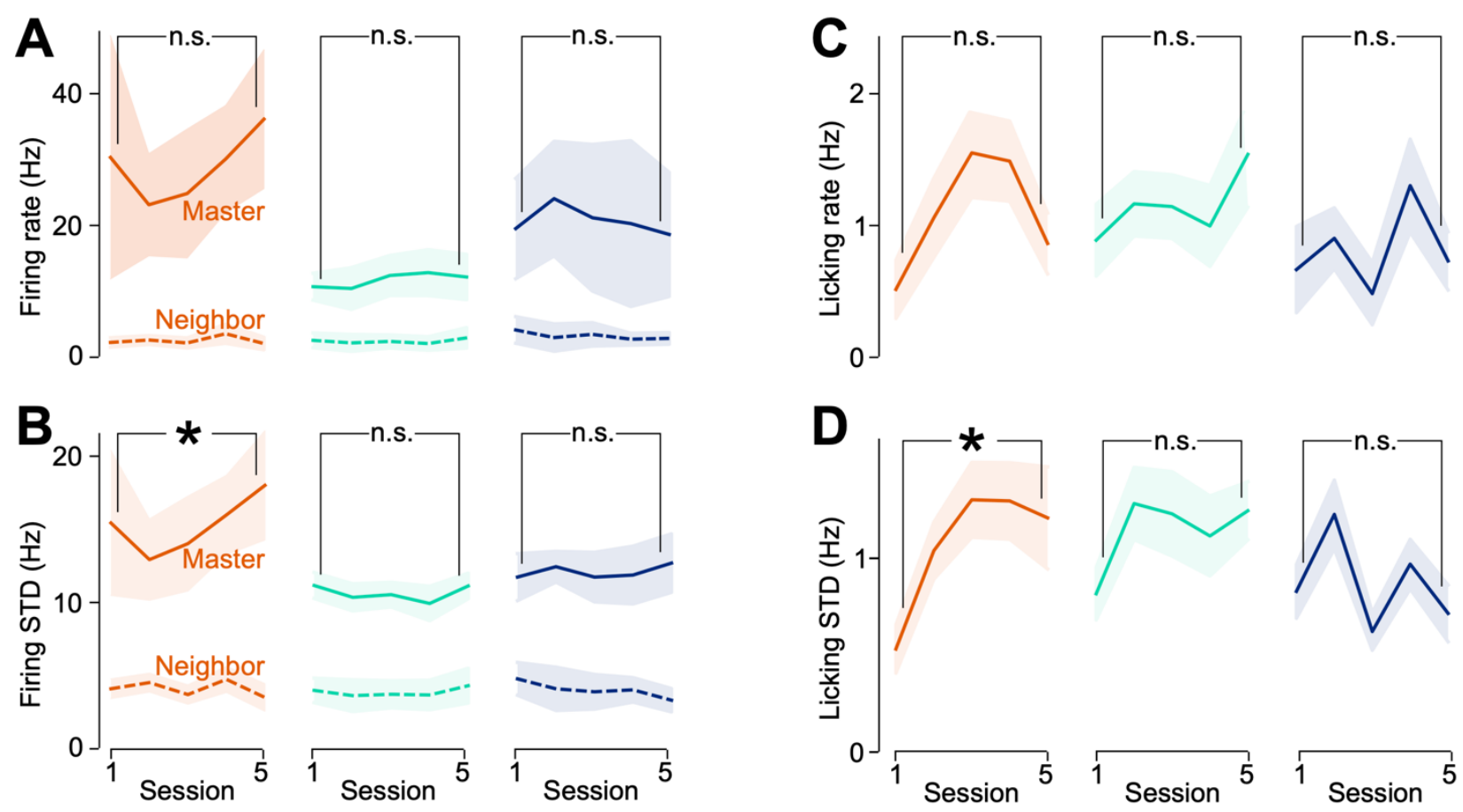

Supplementary Figure 4. Firing statistics of Master and Neighbor neurons, and licking statistics, during closed-loop learning. (A) Average firing rate across all mice of Master (continuous line) and Neighbor neurons (dashed line) over 5 training sessions, during the biomimetic (orange), shuffled (green) and no-feedback (blue) conditions. Shaded background: +/- SEM. (B) Same as (A) for the standard deviation of the firing rate (measured over $1 \mathrm{~s}$ windows). (C) Average licking rate over sessions. (D) Average standard deviation of the licking rate (measured over $1 \mathrm{~s}$ windows). All tests: Mann-Whitney. $*$ : $p<0.05$. 


\section{References}

Abbasi, A., Goueytes, D., Shulz, D.E., Ego-Stengel, V., and Estebanez, L. (2018). A fast intracortical brain-machine interface with patterned optogenetic feedback. J. Neural Eng. 15, 046011.

Andermann, M.L., and Moore, C.I. (2006). A somatotopic map of vibrissa motion direction within a barrel column. Nat. Neurosci. 9, 543-551.

Arduin, P.-J., Fregnac, Y., Shulz, D.E., and Ego-Stengel, V. (2013). "Master" Neurons Induced by Operant Conditioning in Rat Motor Cortex during a Brain-Machine Interface Task. J. Neurosci. 33, 8308-8320.

Arduin, P.-J., Fregnac, Y., Shulz, D.E., and Ego-Stengel, V. (2014). Bidirectional control of a one-dimensional robotic actuator by operant conditioning of a single unit in rat motor cortex. Front. Neurosci. 8, 206.

Armenta Salas, M., Bashford, L., Kellis, S., Jafari, M., Jo, H., Kramer, D., Shanfield, K., Pejsa, K., Lee, B., Liu, C.Y., et al. (2018). Proprioceptive and cutaneous sensations in humans elicited by intracortical microstimulation. ELife 7, e32904.

Athalye, V.R., Ganguly, K., Costa, R.M., and Carmena, J.M. (2017). Emergence of Coordinated Neural Dynamics Underlies Neuroprosthetic Learning and Skillful Control. Neuron 93, 955-970.e5.

Bensmaia, S.J., and Miller, L.E. (2014). Restoring sensorimotor function through intracortical interfaces: progress and looming challenges. Nat. Rev. Neurosci. 15, 313-325.

Brochier, T., Boudreau, M., Pare, M., and Smith, A.M. (1999). The effects of muscimol inactivation of small regions of motor and somatosensory cortex on independent finger movements and force control in the precision grip. Exp. Brain Res. 128, 31-40.

Ceballo, S., Piwkowska, Z., Bourg, J., Daret, A., and Bathellier, B. (2019). Targeted Cortical Manipulation of Auditory Perception. Neuron 104, 1168-1179.

Chesler, A.T., Szczot, M., Bharucha-Goebel, D., Čeko, M., Donkervoort, S., Laubacher, C., Hayes, L.H., Alter, K., Zampieri, C., Stanley, C., Innes, A.M., Mah, J.K., Grosmann, C.M., Bradley, N., Nguyen, D., Foley, A.R., Le Pichon, C.E., and Bönnemann, C.G. (2016) The role of PIEZO2 in human mechanosensation. New England Journal of Medicine. 375, 13551364.

Diamond, M.E., and Arabzadeh, E. (2013). Whisker sensory system - From receptor to decision. Prog. Neurobiol. 103, 28-40.

Estebanez, L., El Boustani, S., Destexhe, A., and Shulz, D.E. (2012). Correlated input reveals coexisting coding schemes in a sensory cortex. Nat. Neurosci. 15, 1691-1699.

Estebanez, L., Bertherat, J., Shulz, D.E., Bourdieu, L., and Léger, J.-F. (2016). A radial map of multi-whisker correlation selectivity in the rat barrel cortex. Nat. Commun. 7, 13528.

Feldmeyer, D. (2012). Excitatory neuronal connectivity in the barrel cortex. Front. Neuroanat. 6.

Ferezou, I., Haiss, F., Gentet, L.J., Aronoff, R., Weber, B., and Petersen, C.C.H. (2007). 
bioRxiv preprint doi: https://doi.org/10.1101/2019.12.12.873794; this version posted July 14,2020 . The copyright holder for this preprint (which was not certified by peer review) is the author/funder, who has granted bioRxiv a license to display the preprint in perpetuity. It is made available under aCC-BY-NC-ND 4.0 International license.

Spatiotemporal Dynamics of Cortical Sensorimotor Integration in Behaving Mice. Neuron 56, 907-923.

Fetz, E.E. (1969). Operant Conditioning of Cortical Unit Activity. Science 163, 955-958.

Flesher, S.N., Collinger, J.L., Foldes, S.T., Weiss, J.M., Downey, J.E., Tyler-Kabara, E.C., Bensmaia, S.J., Schwartz, A.B., Boninger, M.L., and Gaunt, R.A. (2016). Intracortical microstimulation of human somatosensory cortex. Sci. Transl. Med. 8, 361ra141.

Flesher, S.N., Downey, J.E., Weiss, J.M., Hughes, C.L., Herrera, A.J., Tyler-Kabara, E.C., Boninger, M.L., Collinger, J.L., and Gaunt, R.A. (2019). Restored tactile sensation improves neuroprosthetic arm control (Clinical Trials). bioRxiv.

Fu, M., and Zuo, Y. (2011). Experience-dependent structural plasticity in the cortex. Trends Neurosci. 34, 177-187.

Guo, Z.V., Hires, S.A., Li, N., O'Connor, D.H., Komiyama, T., Ophir, E., Huber, D., Bonardi, C., Morandell, K., Gutnisky, D., Peron, S., Xu, N.L., Cox, J., and Svoboda, K. (2014) Procedures for behavioral experiments in head-fixed mice. PLOS ONE 9, e88678.

Hartmann, K., Thomson, E.E., Zea, I., Yun, R., Mullen, P., Canarick, J., Huh, A., and Nicolelis, M.A.L. (2016). Embedding a Panoramic Representation of Infrared Light in the Adult Rat Somatosensory Cortex through a Sensory Neuroprosthesis. J. Neurosci. 36, 2406-2424.

Jacob, V., Le Cam, J., Ego-Stengel, V., and Shulz, D.E. (2008). Emergent Properties of Tactile Scenes Selectively Activate Barrel Cortex Neurons. Neuron 60, 1112-1125.

Johansson, R.S., and Flanagan, J.R. (2009). Coding and use of tactile signals from the fingertips in object manipulation tasks. Nat. Rev. Neurosci. 10, 345-359.

Johansson, R.S., Westling, G. (1984). Roles of glabrous skin receptors and sensorimotor memory in automatic control of precision grip when lifting rougher or more slippery objects.. Exp. Brain Res. 56, 550-564.

Kaas, J.H. (1997). Topographic Maps are Fundamental to Sensory Processing. Brain Res. Bull. 44, 107-112.

Knutsen, P.M., Mateo, C., and Kleinfeld, D. (2016). Precision mapping of the vibrissa representation within murine primary somatosensory cortex. Philos. Trans. R. Soc. B Biol. Sci. 371, 20150351.

Kremer, Y., Leger, J.-F., Goodman, D., Brette, R., and Bourdieu, L. (2011). Late Emergence of the Vibrissa Direction Selectivity Map in the Rat Barrel Cortex. J. Neurosci. 31, 1068910700.

Madisen, L., Mao, T., Koch, H., Zhuo, J., Berenyi, A., Fujisawa, S., Hsu, Y.-W.A., Garcia, A.J., Gu, X., Zanella, S., et al. (2012). A toolbox of Cre-dependent optogenetic transgenic mice for light-induced activation and silencing. Nat. Neurosci. 15, 793-802.

Mathis, A., Mamidanna, P., Cury, K.M., Abe, T., Murthy, V.N., Mathis, M.W., and Bethge, M. (2018). DeepLabCut: markerless pose estimation of user-defined body parts with deep learning. Nat. Neurosci. 21, 1281-1289. 
Mathis, M.W., Mathis, A., and Uchida, N. (2017). Somatosensory Cortex Plays an Essential Role in Forelimb Motor Adaptation in Mice. Neuron 93, 1493-1503.

Miall, R.C., Kitchen, N.M., Nam, S.H., Lefumat, H., Renault, A.G., Ørstavik, K., Cole, J.D., and Sarlegna, F.R. (2018) Proprioceptive loss and the perception, control and learning of arm movements in humans: evidence from sensory neuronopathy. Exp Brain Res. 236, 2137-2155.

Monzée, J., Lamarre, Y., and Smith, A.M. (2003). The Effects of Digital Anesthesia on Force Control Using a Precision Grip. J. Neurophysiol. 89, 672-683.

Moritz, C.T., Perlmutter, S.I., and Fetz, E.E. (2008). Direct control of paralysed muscles by cortical neurons. Nature 456, 639-642.

Oby, E.R., Golub, M.D., Hennig, J.A., Degenhart, A.D., Tyler-Kabara, E.C., Yu, B.M., Chase, S.M., and Batista, A.P. (2019). New neural activity patterns emerge with long-term learning. Proc. Natl. Acad. Sci. 116, 15210-15215.

O'Connor, D.H., Hires, S.A., Guo, Z.V., Li, N., Yu, J., Sun, Q.-Q., Huber, D., and Svoboda, K. (2013). Neural coding during active somatosensation revealed using illusory touch. Nat. Neurosci. 16, 958-965.

O’Doherty, J.E., Lebedev, M.A., Ifft, P.J., Zhuang, K.Z., Shokur, S., Bleuler, H., and Nicolelis, M.A.L. (2011). Active tactile exploration using a brain-machine-brain interface. Nature 479, 228-231.

20 O'Doherty, J.E., Shokur, S., Medina, L.E., Lebedev, M.A., and Nicolelis, M.A.L. (2019). Creating a neuroprosthesis for active tactile exploration of textures. Proc. Natl. Acad. Sci. $116,21821-21827$.

Okun, M., Lak, A., Carandini, M., and Harris, K.D. (2016). Long Term Recordings with Immobile Silicon Probes in the Mouse Cortex. PLOS ONE 11, e0151180.

Papale, A.E., and Hooks, B.M. (2018). Circuit Changes in Motor Cortex During Motor Skill Learning. Neuroscience 368, 283-297.

Penfield, W., and Boldrey, E. (1937). Somatic motor and sensory representation in the cerebral cortex of man as studied by electrical stimulation. Brain $60,389-443$.

Petersen, C.C.H. (2019). Sensorimotor processing in the rodent barrel cortex. Nat. Rev. Neurosci. 20, 533-546.

Prsa, M., Galiñanes, G.L., and Huber, D. (2017). Rapid Integration of Artificial Sensory Feedback during Operant Conditioning of Motor Cortex Neurons. Neuron 93, 929-939.e6.

Romo, R., Hernández, A., Zainos, A., and Salinas, E. (1998). Somatosensory discrimination based on cortical microstimulation. Nature 392, 387-390.

Sadtler, P.T., Quick, K.M., Golub, M.D., Chase, S.M., Ryu, S.I., Tyler-Kabara, E.C., Yu, B.M., and Batista, A.P. (2014). Neural constraints on learning. Nature 512, 423-426.

Sainburg, R., Ghilardi, M., Poizner, H., and Ghez, C. (1995). Control of limb dynamics in normal subjects and patients without proprioception. J. Neurophysiol. 73, 820-835. 
Scott, S.H. (2016). A Functional Taxonomy of Bottom-Up Sensory Feedback Processing for Motor Actions. Trends Neurosci. 39, 512-526.

Simons, D.J. (1978). Response properties of vibrissa units in rat SI somatosensory neocortex. J. Neurophysiol. 41, 798-820.

$5 \quad$ Tabot, G.A., Dammann, J.F., Berg, J.A., Tenore, F.V., Boback, J.L., Vogelstein, R.J., and Bensmaia, S.J. (2013). Restoring the sense of touch with a prosthetic hand through a brain interface. Proc. Natl. Acad. Sci. 110, 18279-18284.

Vilarchao, M.E., Estebanez, L., Shulz, D.E., and Férézou, I. (2018). Supra-barrel Distribution of Directional Tuning for Global Motion in the Mouse Somatosensory Cortex. Cell Rep. 22, 3534-3547.

Woolsey, T.A., and Van der Loos, H. (1970). The structural organization of layer IV in the somatosensory region (SI) of mouse cerebral cortex: the description of a cortical field composed of discrete cytoarchitectonic units. Brain Res. 17, 205-242.

Yger, P., Spampinato, G.L., Esposito, E., Lefebvre, B., Deny, S., Gardella, C., Stimberg, M., Jetter, F., Zeck, G., Picaud, S., et al. (2018). A spike sorting toolbox for up to thousands of electrodes validated with ground truth recordings in vitro and in vivo. ELife 7, e34518. 